\title{
Lone Pair Activity in Lead(II) Complexes with Unsymmetrical Lariat Ethers
}

David Esteban-Gómez, Carlos Platas-Iglesias, Teresa Enríquez-Pérez, Fernando Avecilla, Andrés de Blas* and Teresa Rodríguez-Blas*

Supporting Information

Number of pages 19 
Table S1 Experimental and Calculated (GIAO Method) ${ }^{13} \mathrm{C}$ NMR shifts for the $\left[\mathrm{Pb}\left(\mathrm{L}^{8}-\mathrm{H}\right)\right]^{+}$complex in acetonitrile solution. See Scheme 2 for numbering.

\begin{tabular}{|c|c|c|c|c|c|}
\hline \multicolumn{6}{|c|}{$\left[\mathbf{P b}\left(\mathbf{L}^{8}-\mathbf{H}\right)\right]^{+}$} \\
\hline${ }^{13} \mathrm{C}$ & $\delta_{\mathrm{i}, \exp }{ }^{\mathrm{a}}$ & $\delta_{\mathrm{i}, \mathrm{calc}}$ & ${ }^{13} \mathrm{C}$ & $\delta_{\mathrm{i}, \exp }{ }^{\mathrm{a}}$ & $\delta_{\text {i,calc }}$ \\
\hline $\mathrm{C} 1$ & 169.4 & 175.4 & C17 & 70.4 & 75.7 \\
\hline $\mathrm{C} 2$ & 125.6 & 130.4 & C18 & 70.2 & 73.5 \\
\hline $\mathrm{C} 3$ & 136.8 & 143.7 & C19 & 69.5 & 74.3 \\
\hline $\mathrm{C} 4$ & 115.9 & 122.3 & $\mathrm{C} 20$ & 55.1 & 54.4 \\
\hline $\mathrm{C} 5$ & 138.0 & 145.9 & $\mathrm{C} 21$ & 56.0 & 62.6 \\
\hline C6 & 125.3 & 128.1 & $\mathrm{C} 22$ & 69.3 & 73.7 \\
\hline $\mathrm{C} 7$ & 168.2 & 175.9 & $\mathrm{C} 23$ & 69.9 & 75.6 \\
\hline $\mathrm{C} 8$ & 152.3 & 163.1 & $\mathrm{C} 24$ & 56.4 & 64.1 \\
\hline C9 & 124.1 & 128.6 & $\mathrm{C} 25$ & 59.6 & 66.4 \\
\hline $\mathrm{C} 10$ & 130.6 & 137.1 & $\mathrm{C} 26$ & 126.2 & 141.2 \\
\hline C11 & 127.8 & 133.0 & $\mathrm{C} 27$ & 143.5 & 154.2 \\
\hline C12 & 134.0 & 138.5 & C28 & 120.9 & 127.5 \\
\hline C13 & 131.8 & 141.2 & $\mathrm{C} 29$ & 122.7 & 137.3 \\
\hline C14 & 59.9 & 66.5 & $\mathrm{C} 30$ & $\mathrm{~b}$ & 129.8 \\
\hline C15 & 56.8 & 60.8 & C31 & 134.2 & 140.8 \\
\hline $\mathrm{C} 16$ & 69.5 & 75.4 & & & \\
\hline
\end{tabular}


Table S2 Experimental (X-ray) and calculated (B3LYP/6-31G(d)) bond angles $\left(^{\circ}\right.$ ) of the $\mathrm{Pb}(\mathrm{II})$ coordination environment obtained for the $\left[\mathrm{Pb}\left(\mathrm{L}^{7}\right)\right]^{2+}$ and $\left[\mathrm{Pb}\left(\mathrm{L}^{8}-\mathrm{H}\right)\right]^{+}$ systems. See Figures 1-3 for numbering scheme.

\begin{tabular}{|c|c|c|c|c|c|}
\hline \multicolumn{3}{|c|}{$\left[\mathbf{P b}\left(\mathbf{L}^{7}\right)\right]^{2+}(\mathbf{I})$} & \multicolumn{3}{|c|}{$\left[\mathrm{Pb}\left(\mathrm{L}^{8}-\mathrm{H}\right)\right]^{+}(\mathrm{II})$} \\
\hline & Exp. & Calc. & & Exp. & Calc. \\
\hline $\mathrm{O}(1)-\mathrm{Pb}-\mathrm{N}(4)$ & $76.89(18)$ & 77.74 & $\mathrm{O}(1)-\mathrm{Pb}-\mathrm{N}(4)$ & $132.9(4)$ & 134.32 \\
\hline $\mathrm{O}(1)-\mathrm{Pb}-\mathrm{N}(2)$ & $122.15(19)$ & 122.50 & $\mathrm{O}(1)-\mathrm{Pb}-\mathrm{N}(2)$ & $68.8(5)$ & 67.16 \\
\hline $\mathrm{N}(4)-\mathrm{Pb}-\mathrm{N}(2)$ & $70.59(18)$ & 74.27 & $\mathrm{~N}(4)-\mathrm{Pb}-\mathrm{N}(2)$ & 71.1(4) & 73.85 \\
\hline $\mathrm{O}(1)-\mathrm{Pb}-\mathrm{O}(2)$ & $99.56(18)$ & 101.10 & $\mathrm{O}(1)-\mathrm{Pb}-\mathrm{O}(2)$ & $78.7(4)$ & 73.87 \\
\hline $\mathrm{N}(3)-\mathrm{Pb}-\mathrm{O}(2)$ & $78.00(18)$ & 76.13 & $\mathrm{~N}(3)-\mathrm{Pb}-\mathrm{O}(2)$ & $110.9(4)$ & 113.06 \\
\hline $\mathrm{N}(4)-\mathrm{Pb}-\mathrm{O}(2)$ & $130.97(18)$ & 129.01 & $\mathrm{~N}(4)-\mathrm{Pb}-\mathrm{O}(2)$ & $135.3(4)$ & 136.96 \\
\hline $\mathrm{O}(1)-\mathrm{Pb}-\mathrm{O}(3)$ & $89.5(2)$ & 90.59 & $\mathrm{O}(1)-\mathrm{Pb}-\mathrm{O}(3)$ & $95.7(5)$ & 93.11 \\
\hline $\mathrm{N}(4)-\mathrm{Pb}-\mathrm{O}(3)$ & $164.2(2)$ & 166.62 & $\mathrm{~N}(4)-\mathrm{Pb}-\mathrm{O}(3)$ & $87.5(4)$ & 88.29 \\
\hline $\mathrm{N}(2)-\mathrm{Pb}-\mathrm{O}(3)$ & $111.4(2)$ & 107.47 & $\mathrm{~N}(2)-\mathrm{Pb}-\mathrm{O}(3)$ & $63.1(4)$ & 61.37 \\
\hline $\mathrm{N}(2)-\mathrm{Pb}-\mathrm{O}(2)$ & $137.80(18)$ & 135.37 & $\mathrm{~N}(2)-\mathrm{Pb}-\mathrm{O}(2)$ & $103.9(4)$ & 99.35 \\
\hline $\mathrm{O}(1)-\mathrm{Pb}-\mathrm{N}(3)$ & $172.1(2)$ & 173.94 & $\mathrm{O}(1)-\mathrm{Pb}-\mathrm{N}(3)$ & $118.8(5)$ & 120.19 \\
\hline $\mathrm{N}(4)-\mathrm{Pb}-\mathrm{N}(3)$ & $98.95(19)$ & 99.71 & $\mathrm{~N}(4)-\mathrm{Pb}-\mathrm{N}(3)$ & $83.1(4)$ & 82.54 \\
\hline $\mathrm{N}(2)-\mathrm{Pb}-\mathrm{N}(3)$ & $61.50(18)$ & 61.33 & $\mathrm{~N}(2)-\mathrm{Pb}-\mathrm{N}(3)$ & $145.2(4)$ & 147.59 \\
\hline $\mathrm{N}(1)-\mathrm{Pb}-\mathrm{O}(2)$ & $62.53(17)$ & 62.55 & $\mathrm{~N}(1)-\mathrm{Pb}-\mathrm{O}(2)$ & $57.8(3)$ & 56.88 \\
\hline $\mathrm{O}(3)-\mathrm{Pb}-\mathrm{N}(3)$ & $95.5(2)$ & 92.52 & $\mathrm{O}(3)-\mathrm{Pb}-\mathrm{N}(3)$ & $140.2(4)$ & 140.81 \\
\hline $\mathrm{O}(1)-\mathrm{Pb}-\mathrm{N}(1)$ & $64.9(2)$ & 63.45 & $\mathrm{O}(1)-\mathrm{Pb}-\mathrm{N}(1)$ & $64.6(4)$ & 64.19 \\
\hline $\mathrm{N}(4)-\mathrm{Pb}-\mathrm{N}(1)$ & $72.51(18)$ & 72.52 & $\mathrm{~N}(4)-\mathrm{Pb}-\mathrm{N}(1)$ & $154.3(4)$ & 154.41 \\
\hline $\mathrm{N}(2)-\mathrm{Pb}-\mathrm{N}(1)$ & $138.71(18)$ & 143.67 & $\mathrm{~N}(2)-\mathrm{Pb}-\mathrm{N}(1)$ & $132.3(4)$ & 130.05 \\
\hline $\mathrm{O}(3)-\mathrm{Pb}-\mathrm{O}(2)$ & $58.6(2)$ & 59.26 & $\mathrm{O}(3)-\mathrm{Pb}-\mathrm{O}(2)$ & $54.1(4)$ & 53.67 \\
\hline $\mathrm{O}(3)-\mathrm{Pb}-\mathrm{N}(1)$ & $109.1(2)$ & 108.27 & $\mathrm{O}(3)-\mathrm{Pb}-\mathrm{N}(1)$ & $111.5(4)$ & 110.44 \\
\hline $\mathrm{N}(3)-\mathrm{Pb}-\mathrm{N}(1)$ & $107.59(19)$ & 110.59 & $\mathrm{~N}(3)-\mathrm{Pb}-\mathrm{N}(1)$ & $71.2(3)$ & 71.92 \\
\hline $\mathrm{N}(5)-\mathrm{Pb}-\mathrm{O}(1)$ & $64.2(2)$ & 64.44 & $\mathrm{O}(4)-\mathrm{Pb}-\mathrm{O}(1)$ & $73.3(4)$ & 74.43 \\
\hline $\mathrm{N}(5)-\mathrm{Pb}-\mathrm{N}(4)$ & $101.05(17)$ & 103.10 & $\mathrm{O}(4)-\mathrm{Pb}-\mathrm{N}(4)$ & $76.0(4)$ & 74.84 \\
\hline $\mathrm{N}(5)-\mathrm{Pb}-\mathrm{N}(2)$ & $76.64(19)$ & 74.52 & $\mathrm{O}(4)-\mathrm{Pb}-\mathrm{N}(2)$ & $79.7(5)$ & 77.66 \\
\hline $\mathrm{N}(5)-\mathrm{Pb}-\mathrm{O}(3)$ & $65.3(2)$ & 65.42 & $\mathrm{O}(4)-\mathrm{Pb}-\mathrm{O}(3)$ & $142.6(5)$ & 138.70 \\
\hline $\mathrm{N}(5)-\mathrm{Pb}-\mathrm{N}(3)$ & $123.5(2)$ & 121.61 & $\mathrm{O}(4)-\mathrm{Pb}-\mathrm{N}(3)$ & $71.5(4)$ & 74.90 \\
\hline $\mathrm{N}(5)-\mathrm{Pb}-\mathrm{N}(1)$ & $128.79(19)$ & 127.37 & $\mathrm{O}(4)-\mathrm{Pb}-\mathrm{N}(1)$ & $96.2(4)$ & 99.34 \\
\hline $\mathrm{N}(5)-\mathrm{Pb}-\mathrm{O}(2)$ & 121.63(19) & 122.59 & $\mathrm{O}(4)-\mathrm{Pb}-\mathrm{O}(2)$ & $148.3(4)$ & 146.58 \\
\hline$A F_{i}^{a}$ & & & $A F_{i}^{a}$ & & \\
\hline
\end{tabular}

${ }^{a}$ Agreement factor between the experimental and calculated bond distances: $A F_{i}=$ $\left[\sum(\exp -c a l c d)^{2} / \sum(\exp )^{2}\right]^{1 / 2}$, where exp and calcd denote calculated and experimental values, respectively. 
Figure S1. ${ }^{1} \mathrm{H}$ NMR spectra of complexes $\mathbf{1}$ (top) and $\mathbf{2}$ (bottom) recorded in acetonitrile- $d_{3}$ solution at $298 \mathrm{~K}$.
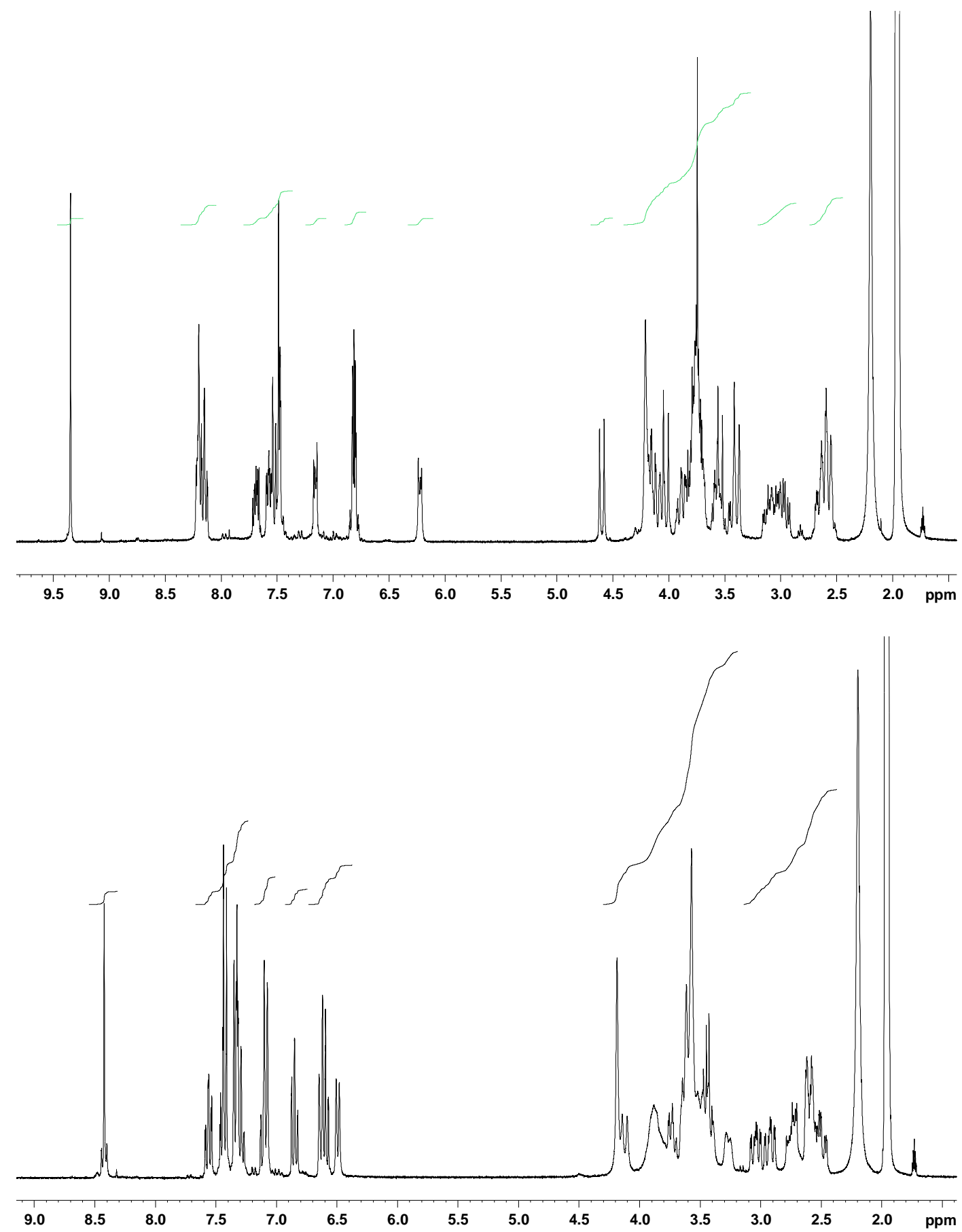


\begin{tabular}{|c|c|c|c|c|}
\hline \multicolumn{5}{|c|}{$(\mathrm{I})-\left[\mathrm{Pb}\left(\mathrm{L}^{7}\right)\right]^{2+} \quad(0$ imaginary frequencies $)$} \\
\hline \multirow{2}{*}{$\begin{array}{l}\text { Center } \\
\text { Number }\end{array}$} & \multirow{2}{*}{$\begin{array}{l}\text { Atomic } \\
\text { Number }\end{array}$} & \multicolumn{3}{|c|}{ Coordinates (Angstroms) } \\
\hline & & $\mathrm{X}$ & $\mathrm{Y}$ & $\mathrm{Z}$ \\
\hline \multicolumn{5}{|c|}{ - - - - - - - - - - - - - - - - - - - - - - - - - - - - - - - - - - - - - - - - - - - - - - - - - - - - - - } \\
\hline 1 & 1 & 0.287904 & -2.366393 & -3.654571 \\
\hline 2 & 1 & 0.129022 & -3.881234 & -2.759780 \\
\hline 3 & 1 & -2.242034 & -2.885885 & -2.909796 \\
\hline 4 & 1 & 1.491953 & -0.641725 & -3.511233 \\
\hline 5 & 1 & 2.473253 & -3.114972 & -2.741043 \\
\hline 6 & 6 & 0.353599 & -2.812705 & -2.654792 \\
\hline 7 & 1 & 3.232321 & -0.737897 & -3.343982 \\
\hline 8 & 6 & -1.921010 & -2.793792 & -1.863869 \\
\hline 9 & 1 & -0.220362 & 0.444958 & -2.819479 \\
\hline 10 & 1 & -1.865514 & -3.807822 & -1.447326 \\
\hline 11 & 6 & 2.313308 & -0.440944 & -2.815604 \\
\hline 12 & 6 & 1.741980 & -2.690428 & -2.032394 \\
\hline 13 & 1 & -5.979842 & 2.346308 & -1.993417 \\
\hline 14 & 8 & -0.621353 & -2.190622 & -1.799926 \\
\hline 15 & 1 & -3.076783 & -0.996190 & -1.712912 \\
\hline 16 & 1 & -3.900221 & -2.447857 & -1.142553 \\
\hline 17 & 1 & -4.259735 & 4.147822 & -1.883297 \\
\hline 18 & 1 & 1.751110 & -3.322032 & -1.141464 \\
\hline 19 & 1 & 4.481309 & 1.192047 & -2.885213 \\
\hline 20 & 6 & 2.373398 & 1.037619 & -2.500545 \\
\hline 21 & 6 & -2.934571 & -1.915406 & -1.139411 \\
\hline 22 & 7 & -0.048122 & 1.077815 & -2.038796 \\
\hline 23 & 1 & -0.823342 & 1.739558 & -2.009309 \\
\hline 24 & 6 & -5.066454 & 2.199186 & -1.426112 \\
\hline 25 & 7 & 2.116533 & -1.319506 & -1.621437 \\
\hline 26 & 6 & 3.585493 & 1.734206 & -2.592810 \\
\hline 27 & 6 & -4.106059 & 3.208011 & -1.361992 \\
\hline 28 & 6 & 1.212435 & 1.761554 & -2.164500 \\
\hline 29 & 1 & 4.219090 & -1.662156 & -1.428720 \\
\hline 30 & 1 & -5.614001 & 0.222543 & -0.784499 \\
\hline 31 & 6 & -4.850419 & 0.995440 & -0.750871 \\
\hline 32 & 6 & 3.662986 & 3.103056 & -2.336195 \\
\hline 33 & 6 & 1.285700 & 3.132778 & -1.905487 \\
\hline 34 & 1 & 4.613497 & 3.619738 & -2.420440 \\
\hline 35 & 6 & 3.367229 & -1.314798 & -0.819144 \\
\hline 36 & 1 & 0.379416 & 3.685354 & -1.668039 \\
\hline 37 & 6 & -2.942524 & 3.010121 & -0.617655 \\
\hline 38 & 6 & 2.507125 & 3.801632 & -1.987668 \\
\hline 39 & 1 & -2.189690 & 3.792677 & -0.568031 \\
\hline 40 & 1 & -2.143111 & -3.557694 & 0.605840 \\
\hline 41 & 7 & -2.550165 & -1.518582 & 0.238884 \\
\hline 42 & 1 & 3.309496 & -3.240274 & 0.211905 \\
\hline 43 & 1 & 3.572054 & -0.274139 & -0.552082 \\
\hline 44 & 1 & 2.548076 & 4.870178 & -1.799800 \\
\hline 45 & 6 & -3.683282 & 0.763265 & -0.013576 \\
\hline 46 & 6 & -2.727820 & 1.800243 & 0.063294 \\
\hline 47 & 82 & 0.029701 & -0.501949 & 0.111287 \\
\hline 48 & 1 & -3.568797 & -2.953443 & 1.451585 \\
\hline 49 & 6 & -2.542066 & -2.698021 & 1.148053 \\
\hline 50 & 6 & 3.352154 & -2.168198 & 0.444164 \\
\hline 51 & 1 & -4.524377 & -1.005188 & 0.830882 \\
\hline 52 & 6 & -3.536973 & -0.522624 & 0.772997 \\
\hline 53 & 1 & 4.292080 & -1.994472 & 0.986169 \\
\hline 54 & 7 & -1.531148 & 1.582268 & 0.793419 \\
\hline 55 & 8 & 2.235822 & -1.815085 & 1.266285 \\
\hline
\end{tabular}




\begin{tabular}{|c|c|c|c|c|}
\hline 56 & 1 & 1.966073 & -3.762686 & 1.929938 \\
\hline 57 & 1 & -3.237927 & -0.294699 & 1.799721 \\
\hline 58 & 8 & -0.325355 & -2.409186 & 1.955572 \\
\hline 59 & 6 & -1.682898 & -2.522569 & 2.393312 \\
\hline 60 & 6 & -1.087674 & 2.501208 & 1.576261 \\
\hline 61 & 1 & -1.668013 & 3.411601 & 1.759910 \\
\hline 62 & 6 & 1.998645 & -2.741230 & 2.332896 \\
\hline 63 & 1 & -1.795079 & -3.411185 & 3.028247 \\
\hline 64 & 1 & -1.968419 & -1.645910 & 2.991196 \\
\hline 65 & 7 & 0.983376 & 1.327973 & 2.008392 \\
\hline 66 & 6 & 0.201277 & 2.400397 & 2.271990 \\
\hline 67 & 6 & 0.670042 & -2.407028 & 2.985261 \\
\hline 68 & 1 & 2.804052 & -2.688293 & 3.078197 \\
\hline 69 & 1 & 0.440581 & -3.171029 & 3.738893 \\
\hline 70 & 1 & -0.071888 & 4.251423 & 3.349831 \\
\hline 71 & 1 & 2.758260 & 0.364561 & 2.391492 \\
\hline 72 & 6 & 2.162918 & 1.241330 & 2.629436 \\
\hline 73 & 6 & 0.585005 & 3.407014 & 3.164190 \\
\hline 74 & 1 & 0.698026 & -1.427257 & 3.480542 \\
\hline 75 & 6 & 2.625463 & 2.204052 & 3.535836 \\
\hline 76 & 6 & 1.820234 & 3.305762 & 3.808797 \\
\hline 77 & 1 & 3.594427 & 2.083383 & 4.008917 \\
\hline 78 & 1 & 2.142020 & 4.072670 & 4.506352 \\
\hline
\end{tabular}

\section{(II)-[Pb(L $\left.\left.\mathbf{L}^{7}\right)\right]^{2+} \quad$ (0 imaginary frequencies)}

\begin{tabular}{|c|c|c|c|c|}
\hline \multirow{2}{*}{$\begin{array}{l}\text { Center } \\
\text { Number }\end{array}$} & \multirow{2}{*}{$\begin{array}{l}\text { Atomic } \\
\text { Number }\end{array}$} & \multicolumn{3}{|c|}{ Coordinates (Angstroms) } \\
\hline & & $\mathrm{X}$ & $\mathrm{Y}$ & $\mathrm{Z}$ \\
\hline \multicolumn{5}{|c|}{ - - - - - - - - - - - - - - - - - - - - - - - - - - - - - - - - - - - - - - - - - - - - - - - - - } \\
\hline 1 & 1 & -5.514449 & -0.014369 & 3.538625 \\
\hline 2 & 1 & -3.637099 & 1.222142 & 4.615594 \\
\hline 3 & 1 & -0.920275 & -2.901260 & 2.259245 \\
\hline 4 & 6 & -4.683227 & 0.348696 & 2.942569 \\
\hline 5 & 1 & -1.440199 & -4.446298 & 1.544032 \\
\hline 6 & 6 & -3.634668 & 1.043767 & 3.544640 \\
\hline 7 & 1 & 1.071082 & -4.264685 & 1.832368 \\
\hline 8 & 1 & -3.359604 & -3.638904 & 0.437891 \\
\hline 9 & 6 & -0.886865 & -3.518810 & 1.350121 \\
\hline 10 & 1 & 4.740757 & -0.378480 & 4.378006 \\
\hline 11 & 1 & -3.155611 & -2.046544 & 1.201455 \\
\hline 12 & 1 & -5.506613 & -0.371315 & 1.091372 \\
\hline 13 & 6 & 0.539005 & -3.855489 & 0.965167 \\
\hline 14 & 6 & -4.669016 & 0.138300 & 1.561815 \\
\hline 15 & 6 & -2.891456 & -2.651586 & 0.328020 \\
\hline 16 & 1 & -1.778464 & 2.095789 & 3.222675 \\
\hline 17 & 6 & -2.588276 & 1.535438 & 2.762864 \\
\hline 18 & 1 & 2.469532 & 0.438506 & 3.843174 \\
\hline 19 & 8 & -1.466303 & -2.810956 & 0.249802 \\
\hline 20 & 6 & 4.452516 & -0.235646 & 3.341098 \\
\hline 21 & 8 & 1.172590 & -2.647634 & 0.515824 \\
\hline 22 & 1 & 0.553842 & -4.594886 & 0.154568 \\
\hline 23 & 1 & -4.462326 & -1.989937 & -0.975950 \\
\hline 24 & 1 & 2.832271 & -3.835699 & 0.103677 \\
\hline 25 & 6 & 3.168950 & 0.219911 & 3.039217 \\
\hline 26 & 6 & -3.362416 & -2.030362 & -0.979944 \\
\hline
\end{tabular}




\begin{tabular}{|c|c|c|c|c|}
\hline 27 & 1 & 6.375544 & -0.809608 & 2.544589 \\
\hline 28 & 1 & 3.157571 & -2.340117 & 0.998168 \\
\hline 29 & 6 & -3.612690 & 0.587759 & 0.760134 \\
\hline 30 & 6 & -2.573100 & 1.318090 & 1.377629 \\
\hline 31 & 6 & 2.570453 & -2.774934 & 0.189083 \\
\hline 32 & 1 & -3.077867 & -2.717660 & -1.781278 \\
\hline 33 & 6 & 5.367643 & -0.479176 & 2.315551 \\
\hline 34 & 1 & 0.960278 & 1.134148 & 2.255001 \\
\hline 35 & 6 & 2.782337 & 0.421160 & 1.710949 \\
\hline 36 & 1 & -4.692820 & 0.247326 & -1.060382 \\
\hline 37 & 6 & -3.651007 & 0.408108 & -0.743317 \\
\hline 38 & 7 & -2.790888 & -0.691121 & -1.281543 \\
\hline 39 & 7 & -1.497964 & 1.827457 & 0.584647 \\
\hline 40 & 7 & 1.457298 & 0.873763 & 1.404682 \\
\hline 41 & 6 & 4.976497 & -0.287680 & 0.990607 \\
\hline 42 & 82 & -0.120115 & -0.553186 & -0.211498 \\
\hline 43 & 1 & -2.076608 & 3.741542 & 1.086388 \\
\hline 44 & 6 & 2.836740 & -2.120584 & -1.165655 \\
\hline 45 & 1 & 2.357009 & -2.739628 & -1.927578 \\
\hline 46 & 6 & 3.681672 & 0.138492 & 0.664335 \\
\hline 47 & 6 & -1.352287 & 3.105036 & 0.564697 \\
\hline 48 & 1 & 3.918442 & -2.148251 & -1.364827 \\
\hline 49 & 1 & 5.688188 & -0.473573 & 0.189775 \\
\hline 50 & 1 & -1.504836 & -2.334427 & -3.200914 \\
\hline 51 & 1 & -3.313316 & 1.332530 & -1.218188 \\
\hline 52 & 1 & 1.442113 & 1.694506 & 0.785122 \\
\hline 53 & 1 & -3.555177 & -0.827968 & -3.286050 \\
\hline 54 & 6 & -2.647032 & -0.512381 & -2.748078 \\
\hline 55 & 7 & 2.317644 & -0.731427 & -1.321647 \\
\hline 56 & 6 & -1.457190 & -1.247276 & -3.343979 \\
\hline 57 & 1 & -1.357300 & 5.708262 & 0.065986 \\
\hline 58 & 1 & 1.045803 & -2.213449 & -3.434510 \\
\hline 59 & 8 & -0.249407 & -0.741626 & -2.738800 \\
\hline 60 & 6 & 3.283273 & 0.295996 & -0.782030 \\
\hline 61 & 6 & -0.294774 & 3.839646 & -0.150221 \\
\hline 62 & 1 & -2.510966 & 0.554177 & -2.948199 \\
\hline 63 & 6 & -0.473477 & 5. 217434 & -0.330249 \\
\hline 64 & 1 & -1.424869 & -1.048517 & -4.422171 \\
\hline 65 & 6 & 0.950852 & -1.119887 & -3.428232 \\
\hline 66 & 1 & 2.804668 & 1.265368 & -0.951901 \\
\hline 67 & 1 & 4.190433 & 0.273422 & -1.402950 \\
\hline 68 & 6 & 2.130383 & -0.421375 & -2.769614 \\
\hline 69 & 7 & 0.795802 & 3.179037 & -0.603542 \\
\hline 70 & 1 & 3.041369 & -0.664510 & -3.337485 \\
\hline 71 & 1 & 0.890195 & -0.785792 & -4.471658 \\
\hline 72 & 1 & 1.973278 & 0.658210 & -2.855403 \\
\hline 73 & 6 & 0.490536 & 5.945026 & -1.029560 \\
\hline 74 & 1 & 0.371466 & 7.012311 & -1.187445 \\
\hline 75 & 6 & 1.713395 & 3.894866 & -1.262204 \\
\hline 76 & 6 & 1.607118 & 5.268657 & -1.510009 \\
\hline 77 & 1 & 2.591364 & 3.354555 & -1.608865 \\
\hline 78 & 1 & 2.391014 & 5.784920 & -2.054284 \\
\hline
\end{tabular}

$\mathrm{HF}=-1669.0987155$ Hartree

Zero-point correction $=0.668053$

Sum of electronic and thermal Free Energies $=-1668.500388$ 
(I)-[Pb( $\left.\left.\mathbf{L}^{8}-\mathbf{H}\right)\right]^{+}$

(0 imaginary frequencies)

\begin{tabular}{|c|c|c|c|c|}
\hline \multirow{2}{*}{$\begin{array}{l}\text { Center } \\
\text { Number }\end{array}$} & \multirow{2}{*}{$\begin{array}{l}\text { Atomic } \\
\text { Number }\end{array}$} & \multicolumn{3}{|c|}{ Coordinates (Angstroms) } \\
\hline & & $\mathrm{X}$ & $\mathrm{Y}$ & $\mathrm{Z}$ \\
\hline-----1 & & --------- & -------- & -------- \\
\hline 1 & 1 & 0.513736 & -3.571215 & -2.463494 \\
\hline 2 & 1 & -0.024073 & -4.786297 & -1.313416 \\
\hline 3 & 1 & -2.113639 & -4.381554 & -0.681256 \\
\hline 4 & 1 & 2.014856 & -2.249852 & -2.687854 \\
\hline 5 & 1 & 2.276786 & -4.363881 & -0.837183 \\
\hline 6 & 6 & 0.304519 & -3.742052 & -1.402384 \\
\hline 7 & 1 & 3.632297 & -2.620544 & -2.122069 \\
\hline 8 & 6 & -1.786089 & -3.428920 & -0.240391 \\
\hline 9 & 1 & 0.580257 & -0.690079 & -2.667933 \\
\hline 10 & 1 & -1.407836 & -3.642343 & 0.770480 \\
\hline 11 & 6 & 2.762435 & -1.965155 & -1.939716 \\
\hline 12 & 6 & 1.561796 & -3.571270 & -0.542280 \\
\hline 13 & 1 & -5.063131 & 0.353773 & -4.207500 \\
\hline 14 & 8 & -0.761009 & -2.856831 & -1.053461 \\
\hline 15 & 1 & -3.159230 & -2.154178 & -1.235057 \\
\hline 16 & 1 & -3.874548 & -3.055338 & 0.106575 \\
\hline 17 & 1 & -3.227968 & 1.976810 & -4.680501 \\
\hline 18 & 1 & 1.280463 & -3.770407 & 0.495527 \\
\hline 19 & 1 & 5.274638 & -0.968273 & -2.116097 \\
\hline 20 & 6 & 3.176156 & -0.525047 & -2.158027 \\
\hline 21 & 6 & -2.984317 & -2.484201 & -0.208195 \\
\hline 22 & 7 & 0.824574 & 0.203451 & -2.246512 \\
\hline 23 & 1 & 0.256106 & 0.922463 & -2.687871 \\
\hline 24 & 6 & -4.346148 & 0.614652 & -3.435287 \\
\hline 25 & 7 & 2.188470 & -2.238117 & -0.596279 \\
\hline 26 & 6 & 4.532197 & -0.180971 & -2.223631 \\
\hline 27 & 6 & -3.321218 & 1.521916 & -3.698574 \\
\hline 28 & 6 & 2.219715 & 0.496228 & -2.329726 \\
\hline 29 & 1 & 4.080430 & -2.818118 & 0.212989 \\
\hline 30 & 1 & -5.271751 & -0.630840 & -1.947258 \\
\hline 31 & 6 & -4.452674 & 0.051611 & -2.162090 \\
\hline 32 & 6 & 4.947457 & 1.134766 & -2.425002 \\
\hline 33 & 6 & 2.633074 & 1.818799 & -2.524645 \\
\hline 34 & 1 & 6.005551 & 1.372455 & -2.470090 \\
\hline 35 & 6 & 3.262915 & -2.097723 & 0.408845 \\
\hline 36 & 1 & 1.886636 & 2.600542 & -2.646646 \\
\hline 37 & 6 & -2.413044 & 1.852989 & -2.692377 \\
\hline 38 & 6 & 3.988737 & 2.137970 & -2.568253 \\
\hline 39 & 1 & -1.617925 & 2.568250 & -2.890006 \\
\hline 40 & 1 & -2.738947 & -2.661616 & 2.234703 \\
\hline 41 & 7 & -2.806192 & -1.285780 & 0.641602 \\
\hline 42 & 1 & 2.549182 & -3.313400 & 2.083866 \\
\hline 43 & 1 & 3.685102 & -1.096157 & 0.292273 \\
\hline 44 & 1 & 4.291442 & 3.169101 & -2.724424 \\
\hline 45 & 6 & -3.546715 & 0.359406 & -1.139947 \\
\hline 46 & 6 & -2.512012 & 1.280955 & -1.413230 \\
\hline 47 & 82 & -0.065772 & -0.282174 & 0.320738 \\
\hline 48 & 1 & -4.083385 & -1.523539 & 2.338029 \\
\hline 49 & 6 & -3.018153 & -1.617244 & 2.070499 \\
\hline 50 & 6 & 2.858602 & -2.279649 & 1.866355 \\
\hline 51 & 1 & -4.788596 & -0.573340 & 0.332314 \\
\hline 52 & 6 & -3.751836 & -0.205633 & 0.248553 \\
\hline 53 & 1 & 3.748085 & -2.077832 & 2.482987 \\
\hline 54 & 7 & -1.559793 & 1.600902 & -0.408122 \\
\hline 55 & 8 & 1.812521 & -1.382643 & 2.206030 \\
\hline 56 & 1 & 1.173436 & -2.560337 & 3.786336 \\
\hline
\end{tabular}




\begin{tabular}{|c|c|c|c|c|}
\hline 57 & 1 & -3.640242 & 0.596959 & 0.984432 \\
\hline 58 & 8 & -0.817622 & -1.133398 & 2.793597 \\
\hline 59 & 6 & -2.178388 & -0.779744 & 3.027417 \\
\hline 60 & 6 & -1.372904 & 2.868719 & -0.143421 \\
\hline 61 & 1 & -2.038225 & 3.583410 & -0.643045 \\
\hline 62 & 6 & 1.390109 & -1.505310 & 3.558877 \\
\hline 63 & 1 & -2.457985 & -1.024649 & 4.062022 \\
\hline 64 & 1 & -2.329995 & 0.300283 & 2.884962 \\
\hline 65 & 6 & 0.583154 & 2.745131 & 1.468792 \\
\hline 66 & 6 & -0.412374 & 3.470653 & 0.730126 \\
\hline 67 & 6 & 0.136821 & -0.673172 & 3.756951 \\
\hline 68 & 1 & 2.178207 & -1.159386 & 4.244505 \\
\hline 69 & 1 & -0.245235 & -0.830384 & 4.774860 \\
\hline 70 & 1 & -1.233829 & 5.419704 & 0.283229 \\
\hline 71 & 1 & 2.223369 & 2.945262 & 2.837586 \\
\hline 72 & 6 & 1.467518 & 3.496809 & 2.287089 \\
\hline 73 & 6 & -0.472044 & 4.887574 & 0.849429 \\
\hline 74 & 1 & 0.343556 & 0.390994 & 3.601786 \\
\hline 75 & 6 & 1.376785 & 4.873045 & 2.378039 \\
\hline 76 & 6 & 0.398714 & 5.587912 & 1.655084 \\
\hline 77 & 1 & 2.072477 & 5.412365 & 3.016022 \\
\hline 78 & 1 & 0.335739 & 6.668256 & 1.732659 \\
\hline 79 & 8 & 0.713776 & 1. 442842 & 1.438204 \\
\hline
\end{tabular}

$\mathrm{HF}=-1727.9724765$ Hartree

Zero-point correction $=0.670124$

Sum of electronic and thermal Free Energies $=-1727.372663$

\section{(II)-[Pb( $\left.\left.\mathrm{L}^{8}-\mathrm{H}\right)\right]^{+} \quad$ (0 imaginary frequencies)}

\begin{tabular}{|c|c|c|c|c|}
\hline \multirow{2}{*}{$\begin{array}{l}\text { Center } \\
\text { Number }\end{array}$} & \multirow{2}{*}{$\begin{array}{l}\text { Atomic } \\
\text { Number }\end{array}$} & \multicolumn{3}{|c|}{ Coordinates (Angstroms) } \\
\hline & & $\mathrm{X}$ & $\mathrm{Y}$ & $\mathrm{Z}$ \\
\hline---- & ---- & ------- & -------- & -------- \\
\hline 1 & 1 & -5.900728 & 0.103897 & 3.419933 \\
\hline 2 & 1 & -4.045035 & 1.299932 & 4.573689 \\
\hline 3 & 1 & -0.989154 & -3.579214 & 2.037098 \\
\hline 4 & 6 & -5.024949 & 0.423622 & 2.863608 \\
\hline 5 & 1 & -1.328371 & -4.981180 & 0.990354 \\
\hline 6 & 6 & -3.987557 & 1.095730 & 3.508525 \\
\hline 7 & 1 & 1.062819 & -4.858252 & 1.309786 \\
\hline 8 & 1 & -3.408655 & -3.690038 & 0.658988 \\
\hline 9 & 6 & -0.872824 & -3.978884 & 1.018463 \\
\hline 10 & 1 & 5.096723 & 0.136005 & 4.319607 \\
\hline 11 & 1 & -2.839715 & -2.138062 & 1.312849 \\
\hline 12 & 1 & -5.765024 & -0.320882 & 0.985508 \\
\hline 13 & 6 & 0.600503 & -4.077373 & 0.686792 \\
\hline 14 & 6 & -4.939127 & 0.173805 & 1.491435 \\
\hline 15 & 6 & -2.830860 & -2.782315 & 0.421752 \\
\hline 16 & 1 & -2.062086 & 2.047760 & 3.274742 \\
\hline 17 & 6 & -2.873091 & 1.518857 & 2.781399 \\
\hline 18 & 1 & 2.872911 & 1.060678 & 3.758442 \\
\hline 19 & 8 & -1.498146 & -3.125890 & 0.070034 \\
\hline 20 & 6 & 4.765793 & 0.148851 & 3.285452 \\
\hline 21 & 8 & 1.199172 & -2.817418 & 0.954736 \\
\hline 22 & 1 & 0.721736 & -4.367170 & -0.368025 \\
\hline 23 & 1 & -4.552356 & -1.959832 & -0.540601 \\
\hline 24 & 1 & 3.072049 & -3.721800 & 0.881913 \\
\hline 25 & 6 & 3.508759 & 0.663797 & 2.969898 \\
\hline 26 & 6 & -3.485673 & -2.093768 & -0.765750 \\
\hline 27 & 1 & 6.591145 & -0.696237 & 2.500937 \\
\hline
\end{tabular}




\begin{tabular}{|c|c|c|c|c|}
\hline 28 & 1 & 3.003693 & -2.042316 & 1.425345 \\
\hline 29 & 6 & -3.822896 & 0.567144 & 0.746384 \\
\hline 30 & 6 & -2.785915 & 1.261584 & 1.408123 \\
\hline 31 & 6 & 2.597790 & -2.745984 & 0.699741 \\
\hline 32 & 1 & -3.436977 & -2.783899 & -1.613912 \\
\hline 33 & 6 & 5.600220 & -0.318063 & 2.270231 \\
\hline 34 & 1 & 1.339588 & 1.621052 & 2.177380 \\
\hline 35 & 6 & 3.061467 & 0.696237 & 1.644853 \\
\hline 36 & 1 & -4.770781 & 0.191604 & -1.142600 \\
\hline 37 & 6 & -3.751240 & 0.341229 & -0.750357 \\
\hline 38 & 7 & -2.877957 & -0.794162 & -1.153565 \\
\hline 39 & 7 & -1.653500 & 1.660620 & 0.647371 \\
\hline 40 & 7 & 1.766005 & 1.225836 & 1.341440 \\
\hline 41 & 6 & 5.147445 & -0.295682 & 0.951079 \\
\hline 42 & 82 & -0.116738 & -0.220483 & -0.106106 \\
\hline 43 & 1 & -2.060525 & 3.639503 & 1.037449 \\
\hline 44 & 6 & 2.916563 & -2.318331 & -0.736870 \\
\hline 45 & 1 & 2.473332 & -3.055528 & -1.411670 \\
\hline 46 & 6 & 3.872550 & 0.177752 & 0.614162 \\
\hline 47 & 6 & -1.407056 & 2.928958 & 0.514740 \\
\hline 48 & 1 & 4.006244 & -2.378604 & -0.890149 \\
\hline 49 & 1 & 5.793305 & -0.664455 & 0.157852 \\
\hline 50 & 1 & -1.582985 & -2.668145 & -2.729862 \\
\hline 51 & 1 & -3.350632 & 1.238925 & -1.228613 \\
\hline 52 & 1 & 1.821322 & 1.977371 & 0.654338 \\
\hline 53 & 1 & -3.507026 & -1.013534 & -3.192646 \\
\hline 54 & 6 & -2.613458 & -0.738745 & -2.605771 \\
\hline 55 & 7 & 2.413908 & -0.975694 & -1.129513 \\
\hline 56 & 6 & -1.458829 & -1.625958 & -3.051563 \\
\hline 57 & 1 & -0.899579 & 5.474278 & 0.477939 \\
\hline 58 & 1 & 0.970933 & -2.767432 & -2.786969 \\
\hline 59 & 8 & -0.238436 & -1.102297 & -2.521282 \\
\hline 60 & 6 & 3.406169 & 0.106713 & -0.823798 \\
\hline 61 & 6 & -0.385260 & 3.529888 & -0.302895 \\
\hline 62 & 1 & -2.356144 & 0.292802 & -2.866175 \\
\hline 63 & 6 & -0.267707 & 4.942877 & -0.230776 \\
\hline 64 & 1 & -1.407256 & -1.607449 & -4.149133 \\
\hline 65 & 6 & 0.937267 & -1.703560 & -3.061587 \\
\hline 66 & 8 & 0.403192 & 1.493777 & -1.388504 \\
\hline 67 & 1 & 2.934637 & 1.043550 & -1.135336 \\
\hline 68 & 1 & 4.293120 & -0.033463 & -1.462800 \\
\hline 69 & 6 & 2.159683 & -0.926360 & -2.592130 \\
\hline 70 & 6 & 0.423064 & 2.799605 & -1.242100 \\
\hline 71 & 1 & 3.036706 & -1.291566 & -3.152978 \\
\hline 72 & 1 & 0.899540 & -1.647002 & -4.158755 \\
\hline 73 & 1 & 1.997713 & 0.120036 & -2.860056 \\
\hline 74 & 6 & 0.610589 & 5.646769 & -1.029319 \\
\hline 75 & 1 & 0.687832 & 6.726080 & -0.952150 \\
\hline 76 & 6 & 1.295198 & 3.558979 & -2.067692 \\
\hline 77 & 6 & 1.391394 & 4.935953 & -1.960061 \\
\hline 78 & 1 & 1.888061 & 3.017038 & -2.798593 \\
\hline 79 & 1 & 2.078577 & 5.473610 & -2.608276 \\
\hline
\end{tabular}

$\mathrm{HF}=-1727.9751446$ Hartree

Zero-point correction $=0.671119$

Sum of electronic and thermal Free Energies = -1727.372374 
(I) $-\left[\mathrm{Pb}\left(\mathrm{L}^{8}\right)\right]^{2+} \quad(0$ imaginary frequencies $)$

\begin{tabular}{|c|c|c|c|c|}
\hline \multirow{2}{*}{$\begin{array}{l}\text { Center } \\
\text { Number }\end{array}$} & \multirow{2}{*}{$\begin{array}{l}\text { Atomic } \\
\text { Number }\end{array}$} & \multicolumn{3}{|c|}{ Coordinates (Angstroms) } \\
\hline & & $\mathrm{X}$ & $\mathrm{Y}$ & $\mathrm{Z}$ \\
\hline & ---- & --------- & --------- & --------- \\
\hline 1 & 1 & 4.328927 & 0.459298 & 1.379549 \\
\hline 2 & 1 & 4.876696 & 0.600778 & -0.295426 \\
\hline 3 & 1 & 3.809416 & 2.895097 & 0.498460 \\
\hline 4 & 1 & 3.017360 & -0.811701 & 2.446533 \\
\hline 5 & 1 & 4.610561 & -1.775940 & 0.355188 \\
\hline 6 & 6 & 4.036232 & 0.287905 & 0.336505 \\
\hline 7 & 1 & 3.296732 & -2.541124 & 2.392621 \\
\hline 8 & 6 & 3.168375 & 2.456796 & -0.277548 \\
\hline 9 & 1 & 1.457957 & 0.684910 & 2.636126 \\
\hline 10 & 1 & 3.722063 & 2.498135 & -1.224239 \\
\hline 11 & 6 & 2.580103 & -1.755718 & 2.107063 \\
\hline 12 & 6 & 3.741110 & -1.179795 & 0.029935 \\
\hline 13 & 1 & -1.411271 & 5.472341 & 3.127577 \\
\hline 14 & 8 & 2.879185 & 1.087726 & 0.037093 \\
\hline 15 & 1 & 1.479739 & 3.358164 & 0.693839 \\
\hline 16 & 1 & 2.099564 & 4.264840 & -0.687578 \\
\hline 17 & 1 & -2.720205 & 3.529348 & 3.985770 \\
\hline 18 & 1 & 3.672254 & -1.276701 & -1.055950 \\
\hline 19 & 1 & 1.736923 & -4.037490 & 3.307417 \\
\hline 20 & 6 & 1.262215 & -2.002072 & 2.807954 \\
\hline 21 & 6 & 1.865912 & 3. 251634 & -0.322983 \\
\hline 22 & 7 & 0.542440 & 0.319789 & 2.373160 \\
\hline 23 & 1 & -0.154644 & 0.991442 & 2.696278 \\
\hline 24 & 6 & -1.523385 & 4.575867 & 2.526380 \\
\hline 25 & 7 & 2.481619 & -1.689783 & 0.614942 \\
\hline 26 & 6 & 0.987112 & -3.253220 & 3.376320 \\
\hline 27 & 6 & -2.256432 & 3.490319 & 3.004717 \\
\hline 28 & 6 & 0.301204 & -0.981575 & 2.947386 \\
\hline 29 & 1 & 2.900612 & -3.777975 & 0.419200 \\
\hline 30 & 1 & -0.406757 & 5.369614 & 0.872458 \\
\hline 31 & 6 & -0.946388 & 4.507685 & 1.257235 \\
\hline 32 & 6 & -0.215118 & -3.507864 & 4.035676 \\
\hline 33 & 6 & -0.906649 & -1.233927 & 3.603328 \\
\hline 34 & 1 & -0.401469 & -4.484836 & 4.469699 \\
\hline 35 & 6 & 2.141259 & -3.042682 & 0.101547 \\
\hline 36 & 1 & -1.634055 & -0.434094 & 3.717083 \\
\hline 37 & 6 & -2.405426 & 2.349990 & 2.212745 \\
\hline 38 & 6 & -1.167174 & -2.494030 & 4.141683 \\
\hline 39 & 1 & -2.979409 & 1.503018 & 2.579343 \\
\hline 40 & 1 & 2.214629 & 2.426431 & -2.682207 \\
\hline 41 & 7 & 0.789550 & 2.630453 & -1.137718 \\
\hline 42 & 1 & 2.966692 & -3.014696 & -1.918734 \\
\hline 43 & 1 & 1.201130 & -3.336769 & 0.576630 \\
\hline 44 & 1 & -2.103949 & -2.673352 & 4.660186 \\
\hline 45 & 6 & -1.067263 & 3.368667 & 0.448959 \\
\hline 46 & 6 & -1.822237 & 2.277808 & 0.936143 \\
\hline 47 & 82 & 0.506283 & 0.115079 & -0.274562 \\
\hline 48 & 1 & 0.966003 & 3.628041 & -3.017283 \\
\hline 49 & 6 & 1.146875 & 2.632797 & -2.584476 \\
\hline 50 & 6 & 2.010072 & -3.185709 & -1.410975 \\
\hline 51 & 1 & -0.340653 & 4.426145 & -1.263820 \\
\hline 52 & 6 & -0.498158 & 3.382803 & -0.953016 \\
\hline 53 & 1 & 1.709177 & -4.218553 & -1.631583 \\
\hline 54 & 7 & -1.950285 & 1.089660 & 0.156445 \\
\hline 55 & 8 & 1.033666 & -2.268089 & -1.929010 \\
\hline 56 & 1 & 2.220586 & -1.856559 & -3.580759 \\
\hline
\end{tabular}




\begin{tabular}{|c|c|c|c|c|}
\hline 57 & 1 & -1.221249 & 2.948255 & -1.647669 \\
\hline 58 & 8 & 0.829549 & 0.298074 & -2.950071 \\
\hline 59 & 6 & 0.410957 & 1.587687 & -3.411968 \\
\hline 60 & 6 & -3.156100 & 0.643235 & -0.023325 \\
\hline 61 & 1 & -3.983383 & 1.258734 & 0.348352 \\
\hline 62 & 6 & 1.164073 & -2.030847 & -3.340755 \\
\hline 63 & 1 & 0.690979 & 1.713134 & -4.466152 \\
\hline 64 & 1 & -0.681476 & 1.680453 & -3.341894 \\
\hline 65 & 6 & -2.797988 & -1.637417 & -1.135740 \\
\hline 66 & 6 & -3.609349 & -0.575631 & -0.673363 \\
\hline 67 & 6 & 0.353541 & -0.807238 & -3.725424 \\
\hline 68 & 1 & 0.818514 & -2.902720 & -3.912270 \\
\hline 69 & 1 & 0.505319 & -0.611318 & -4.794929 \\
\hline 70 & 1 & -5.649313 & 0.093100 & -0.478240 \\
\hline 71 & 1 & -2.721348 & -3.573377 & -2.069864 \\
\hline 72 & 6 & -3.362541 & -2.762319 & -1.732240 \\
\hline 73 & 6 & -5.008612 & -0.708802 & -0.835119 \\
\hline 74 & 1 & -0.720562 & -0.956904 & -3.553798 \\
\hline 75 & 6 & -4.746489 & -2.853437 & -1.885363 \\
\hline 76 & 6 & -5.577098 & -1.822655 & -1.435959 \\
\hline 77 & 1 & -5.173437 & -3.735715 & -2.352211 \\
\hline 78 & 1 & -6.653231 & -1.893828 & -1.550277 \\
\hline 79 & 8 & -1.437753 & -1.537418 & -0.976070 \\
\hline 80 & 1 & -0.956863 & -2.286809 & -1.378630 \\
\hline
\end{tabular}

$\mathrm{HF}=-1728.2883362$ Hartree

Zero-point correction $=0.684457$

Sum of electronic and thermal Free Energies = -1727.672334

\section{(II)-[ $\left.\mathrm{Pb}\left(\mathrm{L}^{8}\right)\right]^{2+} \quad(0$ imaginary frequencies $)$}

\begin{tabular}{|c|c|c|c|c|}
\hline \multirow{2}{*}{$\begin{array}{l}\text { Center } \\
\text { Number }\end{array}$} & \multirow{2}{*}{$\begin{array}{l}\text { Atomic } \\
\text { Number }\end{array}$} & \multicolumn{3}{|c|}{ Coordinates (Angstroms) } \\
\hline & & $\mathrm{X}$ & $\mathrm{Y}$ & Z \\
\hline-----1 & ------ & ------- & -------- & -------- \\
\hline 1 & 1 & -5.693858 & 0.426617 & 3.478035 \\
\hline 2 & 1 & -3.728518 & 1.404156 & 4.652531 \\
\hline 3 & 1 & -1.356684 & -3.212038 & 2.193086 \\
\hline 4 & 6 & -4.803651 & 0.699646 & 2.920349 \\
\hline 5 & 1 & -1.852369 & -4.586946 & 1.173946 \\
\hline 6 & 6 & -3.704176 & 1.251191 & 3.577971 \\
\hline 7 & 1 & 0.578258 & -4.662839 & 1.650206 \\
\hline 8 & 1 & -3.701974 & -3.380257 & 0.388562 \\
\hline 9 & 6 & -1.261627 & -3.661423 & 1.194488 \\
\hline 10 & 1 & 4.933092 & -0.258034 & 4.345022 \\
\hline 11 & 1 & -3.311486 & -1.849446 & 1.201654 \\
\hline 12 & 1 & -5.636098 & 0.114952 & 1.024361 \\
\hline 13 & 6 & 0.183490 & -3.979555 & 0.887662 \\
\hline 14 & 6 & -4.763039 & 0.513194 & 1.535797 \\
\hline 15 & 6 & -3.125718 & -2.448852 & 0.303144 \\
\hline 16 & 1 & -1.718742 & 2.070428 & 3.355037 \\
\hline 17 & 6 & -2.572970 & 1.626915 & 2.850238 \\
\hline 18 & 1 & 2.720348 & 0.688745 & 3.775703 \\
\hline 19 & 8 & -1.730818 & -2.754695 & 0.192487 \\
\hline 20 & 6 & 4.619764 & -0.212434 & 3.306680 \\
\hline 21 & 8 & 0.921113 & -2.751373 & 0.901078 \\
\hline 22 & 1 & 0.263603 & -4.465311 & -0.094536 \\
\hline 23 & 1 & -4.633489 & -1.559422 & -0.936259 \\
\hline 24 & 1 & 2.636888 & -3.931954 & 0.816476 \\
\hline 25 & 6 & 3.368198 & 0.315552 & 2.985461 \\
\hline 26 & 6 & -3.548454 & -1.733505 & -0.970276 \\
\hline
\end{tabular}




\begin{tabular}{|c|c|c|c|c|}
\hline 27 & 1 & 6.455546 & -1.040053 & 2.530624 \\
\hline 28 & 1 & 2.842261 & -2.274498 & 1.387660 \\
\hline 29 & 6 & -3.628984 & 0.849572 & 0.788782 \\
\hline 30 & 6 & -2.535138 & 1.434429 & 1.464402 \\
\hline 31 & 6 & 2.328880 & -2.892433 & 0.653895 \\
\hline 32 & 1 & -3.369952 & -2.421512 & -1.800458 \\
\hline 33 & 6 & 5.470631 & -0.651757 & 2.292390 \\
\hline 34 & 1 & 1.184076 & 1. 224651 & 2.189125 \\
\hline 35 & 6 & 2.950759 & 0.388395 & 1.654560 \\
\hline 36 & 1 & -4.639245 & 0.656294 & -1.095158 \\
\hline 37 & 6 & -3.606380 & 0.711266 & -0.720020 \\
\hline 38 & 7 & -2.826610 & -0.458168 & -1.237703 \\
\hline 39 & 7 & -1.398126 & 1.800237 & 0.684038 \\
\hline 40 & 7 & 1.652433 & 0.929513 & 1.332883 \\
\hline 41 & 6 & 5.045714 & -0.587648 & 0.965099 \\
\hline 42 & 82 & -0.193863 & -0.480037 & -0.121444 \\
\hline 43 & 1 & -1.583253 & 3.762340 & 1.287798 \\
\hline 44 & 6 & 2.682398 & -2.504905 & -0.782308 \\
\hline 45 & 1 & 2.175056 & -3.204133 & -1.453522 \\
\hline 46 & 6 & 3.778933 & -0.094532 & 0.623763 \\
\hline 47 & 6 & -1.040444 & 3.038818 & 0.667020 \\
\hline 48 & 1 & 3.762502 & -2.650927 & -0.934134 \\
\hline 49 & 1 & 5.707842 & -0.932428 & 0.174731 \\
\hline 50 & 1 & -1.726891 & -2.254433 & -3.080281 \\
\hline 51 & 1 & -3.155325 & 1.607310 & -1.152957 \\
\hline 52 & 1 & 1.745978 & 1.774701 & 0.766023 \\
\hline 53 & 1 & -3.536263 & -0.459577 & -3.265017 \\
\hline 54 & 6 & -2.609010 & -0.286405 & -2.695679 \\
\hline 55 & 7 & 2.288919 & -1.123089 & -1.188285 \\
\hline 56 & 6 & -1.529920 & -1.192327 & -3.269843 \\
\hline 57 & 1 & -0.026087 & 5.445996 & 0.982590 \\
\hline 58 & 1 & 0.680864 & -2.690555 & -2.912510 \\
\hline 59 & 8 & -0.252197 & -0.843424 & -2.693187 \\
\hline 60 & 6 & 3.340632 & -0.104478 & -0.821363 \\
\hline 61 & 6 & 0.017552 & 3.619391 & -0.157494 \\
\hline 62 & 1 & -2.316223 & 0.752431 & -2.875030 \\
\hline 63 & 6 & 0.420468 & 4.941496 & 0.129904 \\
\hline 64 & 1 & -1.478027 & -1.045701 & -4.356011 \\
\hline 65 & 6 & 0.834067 & -1.641371 & -3.196818 \\
\hline 66 & 8 & 0.192042 & 1.701632 & -1.601008 \\
\hline 67 & 1 & 2.935175 & 0.870201 & -1.117595 \\
\hline 68 & 1 & 4.227208 & -0.279589 & -1.448287 \\
\hline 69 & 6 & 2.142936 & -1.074622 & -2.669000 \\
\hline 70 & 6 & 0.587008 & 2.993153 & -1.289975 \\
\hline 71 & 1 & 2.976458 & -1.599397 & -3.160400 \\
\hline 72 & 1 & 0.842020 & -1.590127 & -4.293020 \\
\hline 73 & 1 & 2.211621 & -0.027312 & -2.980594 \\
\hline 74 & 6 & 1.355217 & 5.608368 & -0.652587 \\
\hline 75 & 1 & 1.648541 & 6.623343 & -0.407162 \\
\hline 76 & 6 & 1.511953 & 3.662215 & -2.088296 \\
\hline 77 & 6 & 1.896296 & 4.966459 & -1.769647 \\
\hline 78 & 1 & 1.925966 & 3.171844 & -2.966644 \\
\hline 79 & 1 & 2.614955 & 5.479315 & -2.401189 \\
\hline 80 & 1 & 0.403259 & 1.486782 & -2.525263 \\
\hline
\end{tabular}

$\mathrm{HF}=-1728.2756036$ Hartree

Zero-point correction $=0.683793$

Sum of electronic and thermal Free Energies $=-1727.660571$ 
(0 imaginary frequencies)

\begin{tabular}{|c|c|c|c|c|}
\hline \multirow{2}{*}{$\begin{array}{l}\text { Center } \\
\text { Number }\end{array}$} & \multirow{2}{*}{$\begin{array}{l}\text { Atomic } \\
\text { Number }\end{array}$} & \multicolumn{3}{|c|}{ Coordinates (Angstroms) } \\
\hline & & $\mathrm{X}$ & $\mathrm{Y}$ & $\mathrm{Z}$ \\
\hline \multicolumn{5}{|c|}{ - - - - - - - - - - - - - - - - - - - - - - - - - - - - - - - - - - - - - - - - - - - - - - - - - - - - - - } \\
\hline 1 & 1 & -4.108053 & -0.767457 & 1.961322 \\
\hline 2 & 1 & -4.717488 & -1.617352 & 0.545357 \\
\hline 3 & 1 & -3.411833 & -3.547746 & 1.034871 \\
\hline 4 & 1 & -3.141714 & 1.008782 & 2.387596 \\
\hline 5 & 1 & -4.961034 & 0.752706 & 0.170651 \\
\hline 6 & 6 & -3.909968 & -0.964967 & 0.900600 \\
\hline 7 & 1 & -3.935201 & 2.452548 & 1.780639 \\
\hline 8 & 6 & -2.730466 & -2.991348 & 0.374430 \\
\hline 9 & 1 & -1.214406 & 0.147669 & 2.550522 \\
\hline 10 & 1 & -3.143824 & -3.044485 & -0.643374 \\
\hline 11 & 6 & -3.013834 & 1.850207 & 1.697286 \\
\hline 12 & 6 & -3.954601 & 0.307585 & 0.051573 \\
\hline 13 & 1 & 1.793318 & -4.255840 & 4.548622 \\
\hline 14 & 8 & -2.647121 & -1.629805 & 0.793375 \\
\hline 15 & 1 & -1.008333 & -3.512309 & 1.499655 \\
\hline 16 & 1 & -1.499047 & -4.739459 & 0.328026 \\
\hline 17 & 1 & 2.672587 & -1.945503 & 4.902908 \\
\hline 18 & 1 & -3.856548 & -0.003514 & -0.990905 \\
\hline 19 & 1 & -2.927421 & 4.535593 & 2.067730 \\
\hline 20 & 6 & -1.834069 & 2.690838 & 2.133414 \\
\hline 21 & 6 & -1.361137 & -3.653188 & 0.475612 \\
\hline 22 & 7 & -0.403345 & 0.694381 & 2.268012 \\
\hline 23 & 1 & 0.403809 & 0.350059 & 2.783512 \\
\hline 24 & 6 & 1.838307 & -3.550357 & 3.724794 \\
\hline 25 & 7 & -2.893172 & 1.292400 & 0.325537 \\
\hline 26 & 6 & -1.964093 & 4.077296 & 2.278513 \\
\hline 27 & 6 & 2.329919 & -2.261077 & 3.921706 \\
\hline 28 & 6 & -0.590612 & 2.100110 & 2.433115 \\
\hline 29 & 1 & -3.793767 & 3.050809 & -0.498149 \\
\hline 30 & 1 & 1.061671 & -4.949080 & 2.291072 \\
\hline 31 & 6 & 1.415768 & -3.933387 & 2.451262 \\
\hline 32 & 6 & -0.895141 & 4.874377 & 2.685891 \\
\hline 33 & 6 & 0.485096 & 2.897433 & 2.837753 \\
\hline 34 & 1 & -1.022224 & 5.947450 & 2.787907 \\
\hline 35 & 6 & -2.906197 & 2.407857 & -0.647621 \\
\hline 36 & 1 & 1.442568 & 2.432387 & 3.061087 \\
\hline 37 & 6 & 2.394372 & -1.374262 & 2.846475 \\
\hline 38 & 6 & 0.335537 & 4.277396 & 2.960218 \\
\hline 39 & 1 & 2.794309 & -0.373007 & 2.988165 \\
\hline 40 & 1 & -1.634965 & -3.702449 & -1.983476 \\
\hline 41 & 7 & -0.328018 & -3.123263 & -0.438260 \\
\hline 42 & 1 & -3.867790 & 1.562623 & -2.415963 \\
\hline 43 & 1 & -2.025955 & 3.024137 & -0.446036 \\
\hline 44 & 1 & 1.180000 & 4.881624 & 3.278077 \\
\hline 45 & 6 & 1.451625 & -3.055125 & 1.359480 \\
\hline 46 & 6 & 1.960839 & -1.754470 & 1.563950 \\
\hline 47 & 82 & -0.318985 & -0.168193 & -0.328355 \\
\hline 48 & 1 & -0.088098 & -4.549264 & -2.010096 \\
\hline 49 & 6 & -0.561056 & -3.569340 & -1.830509 \\
\hline 50 & 6 & -2.904570 & 2.001972 & -2.116776 \\
\hline 51 & 1 & 1.101807 & -4.647502 & -0.025776 \\
\hline 52 & 6 & 1.032636 & -3.546183 & -0.009914 \\
\hline 53 & 1 & -2.760522 & 2.909124 & -2.721666 \\
\hline 54 & 7 & 2.031090 & -0.812952 & 0.492459 \\
\hline 55 & 8 & -1.859419 & 1.072330 & -2.367149 \\
\hline 56 & 1 & -2.857068 & 0.047564 & -3.861540 \\
\hline
\end{tabular}




\begin{tabular}{|c|c|c|c|c|}
\hline 57 & 1 & 1.734547 & -3.173079 & -0.761803 \\
\hline 58 & 8 & -0.889609 & -1.411793 & -2.769256 \\
\hline 59 & 6 & -0.083806 & -2.580901 & -2.887738 \\
\hline 60 & 6 & 3.220611 & -0.321878 & 0.275002 \\
\hline 61 & 1 & 4.033274 & -0.772359 & 0.855734 \\
\hline 62 & 6 & -1.886787 & 0.537029 & -3.687202 \\
\hline 63 & 1 & -0.218637 & -3.031252 & -3.882039 \\
\hline 64 & 1 & 0.982024 & -2.330373 & -2.778525 \\
\hline 65 & 6 & 2.931842 & 1.608000 & -1.422061 \\
\hline 66 & 6 & 3.695218 & 0.721510 & -0.602568 \\
\hline 67 & 6 & -0.761293 & -0.470396 & -3.836861 \\
\hline 68 & 1 & -1.761752 & 1.336379 & -4.431640 \\
\hline 69 & 1 & -0.863292 & -0.978684 & -4.806431 \\
\hline 70 & 1 & 5.685411 & 0.182446 & 0.049447 \\
\hline 71 & 1 & 3.067503 & 3.260886 & -2.785605 \\
\hline 72 & 6 & 3.641235 & 2.579448 & -2.165867 \\
\hline 73 & 6 & 5.113411 & 0.859259 & -0.580874 \\
\hline 74 & 1 & 0.215645 & 0.025911 & -3.792321 \\
\hline 75 & 6 & 5.022682 & 2.681848 & -2.126651 \\
\hline 76 & 6 & 5.777917 & 1.810511 & -1.326190 \\
\hline 77 & 1 & 5.518826 & 3.445449 & -2.719626 \\
\hline 78 & 1 & 6.859855 & 1.885455 & -1.288715 \\
\hline 79 & 16 & 1.188473 & 1.647397 & -1.642222 \\
\hline
\end{tabular}

$\mathrm{HF}=-2050.9290483$ Hartree

Zero-point correction $=0.668884$

Sum of electronic and thermal Free Energies = -2050.330295

\section{(II)-[Pb( $\left.\left.\mathrm{L}^{9}-\mathrm{H}\right)\right]^{+} \quad$ (0 imaginary frequencies)}

\begin{tabular}{|c|c|c|c|c|}
\hline \multirow{2}{*}{$\begin{array}{l}\text { Center } \\
\text { Number }\end{array}$} & \multirow{2}{*}{$\begin{array}{l}\text { Atomic } \\
\text { Number }\end{array}$} & \multicolumn{3}{|c|}{ Coordinates (Angstroms) } \\
\hline & & $\mathrm{X}$ & $\mathrm{Y}$ & $\mathrm{Z}$ \\
\hline \multicolumn{5}{|c|}{ 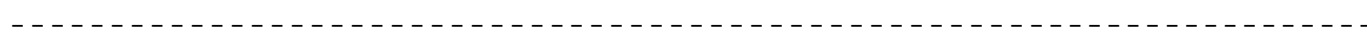 } \\
\hline 1 & 1 & -5.982919 & 0.530090 & 3.216036 \\
\hline 2 & 1 & -4.118924 & 1.669841 & 4.415625 \\
\hline 3 & 1 & -1.269498 & -3.159417 & 2.493696 \\
\hline 4 & 6 & -5.066657 & 0.783570 & 2.691673 \\
\hline 5 & 1 & -1.717168 & -4.689543 & 1.695366 \\
\hline 6 & 6 & -4.025684 & 1.425603 & 3.361495 \\
\hline 7 & 1 & 0.678058 & -4.703303 & 2.062721 \\
\hline 8 & 1 & -3.678382 & -3.396918 & 0.984156 \\
\hline 9 & 6 & -1.174369 & -3.740393 & 1.563829 \\
\hline 10 & 1 & 5.075939 & 0.346788 & 4.207484 \\
\hline 11 & 1 & -3.052318 & -1.813059 & 1.489511 \\
\hline 12 & 1 & -5.753160 & -0.002702 & 0.811810 \\
\hline 13 & 6 & 0.287139 & -4.020969 & 1.292588 \\
\hline 14 & 6 & -4.928703 & 0.473964 & 1.336687 \\
\hline 15 & 6 & -3.030443 & -2.558135 & 0.682353 \\
\hline 16 & 1 & -2.049855 & 2.277891 & 3.182855 \\
\hline 17 & 6 & -2.863089 & 1.770779 & 2.671025 \\
\hline 18 & 1 & 2.957543 & 1.361246 & 3.433060 \\
\hline 19 & 8 & -1.704394 & -3.016174 & 0.463415 \\
\hline 20 & 6 & 4.760038 & 0.210141 & 3.177497 \\
\hline 21 & 8 & 0.987916 & -2.786206 & 1.328648 \\
\hline 22 & 1 & 0.389880 & -4.514717 & 0.314524 \\
\hline 23 & 1 & -4.632109 & -1.774287 & -0.506299 \\
\hline 24 & 1 & 2.774934 & -3.847614 & 1.413632 \\
\hline 25 & 6 & 3.561992 & 0.776350 & 2.743003 \\
\hline 26 & 6 & -3.561396 & -1.990470 & -0.625182 \\
\hline 27 & 1 & 6.505707 & -0.923461 & 2.602781 \\
\hline
\end{tabular}




\begin{tabular}{|c|c|c|c|c|}
\hline 28 & 1 & 2.858691 & -2.100413 & 1.659651 \\
\hline 29 & 6 & -3.759689 & 0.777672 & 0.630003 \\
\hline 30 & 6 & -2.728014 & 1.459649 & 1.311861 \\
\hline 31 & 6 & 2.384174 & -2.879911 & 1.066049 \\
\hline 32 & 1 & -3.490905 & -2.785399 & -1.372657 \\
\hline 33 & 6 & 5.558423 & -0.501544 & 2.282040 \\
\hline 34 & 1 & 1.527213 & 1.817400 & 1.710236 \\
\hline 35 & 6 & 3.136948 & 0.619986 & 1.419370 \\
\hline 36 & 1 & -4.637711 & 0.362147 & -1.281467 \\
\hline 37 & 6 & -3.629723 & 0.452741 & -0.844568 \\
\hline 38 & 7 & -2.848125 & -0.786260 & -1.128791 \\
\hline 39 & 7 & -1.554202 & 1.809667 & 0.586725 \\
\hline 40 & 7 & 1.907508 & 1.208692 & 0.988533 \\
\hline 41 & 6 & 5.125912 & -0.667392 & 0.966253 \\
\hline 42 & 82 & -0.151856 & -0.303018 & -0.145878 \\
\hline 43 & 1 & -1.872402 & 3.776918 & 1.066655 \\
\hline 44 & 6 & 2.717986 & -2.735416 & -0.422558 \\
\hline 45 & 1 & 2.187606 & -3.524962 & -0.962505 \\
\hline 46 & 6 & 3.906664 & -0.143182 & 0.517475 \\
\hline 47 & 6 & -1.242933 & 3.061241 & 0.522229 \\
\hline 48 & 1 & 3.792827 & -2.936363 & -0.564143 \\
\hline 49 & 1 & 5.742685 & -1.226822 & 0.266918 \\
\hline 50 & 1 & -1.782693 & -2.921912 & -2.588424 \\
\hline 51 & 1 & -3.122439 & 1.273101 & -1.358588 \\
\hline 52 & 1 & 2.038273 & 1.786353 & 0.157969 \\
\hline 53 & 1 & -3.532732 & -1.138996 & -3.129838 \\
\hline 54 & 6 & -2.605952 & -0.888847 & -2.585685 \\
\hline 55 & 7 & 2.352887 & -1.433882 & -1.040562 \\
\hline 56 & 6 & -1.545888 & -1.916467 & -2.961105 \\
\hline 57 & 1 & -0.397932 & 5.428721 & 1.018116 \\
\hline 58 & 1 & 0.733087 & -3.313667 & -2.442538 \\
\hline 59 & 8 & -0.280091 & -1.503100 & -2.438871 \\
\hline 60 & 6 & 3.447650 & -0.417411 & -0.897501 \\
\hline 61 & 6 & -0.151037 & 3.679495 & -0.218822 \\
\hline 62 & 1 & -2.267969 & 0.089119 & -2.940873 \\
\hline 63 & 6 & 0.154710 & 5.002158 & 0.184000 \\
\hline 64 & 1 & -1.482306 & -1.965053 & -4.056965 \\
\hline 65 & 6 & 0.820557 & -2.304214 & -2.868663 \\
\hline 66 & 16 & 0.243879 & 1.526505 & -2.076144 \\
\hline 67 & 1 & 3.075175 & 0.494201 & -1.378492 \\
\hline 68 & 1 & 4.320628 & -0.750593 & -1.481952 \\
\hline 69 & 6 & 2.122752 & -1.608293 & -2.497047 \\
\hline 70 & 6 & 0.537959 & 3.113545 & -1.334112 \\
\hline 71 & 1 & 2.954870 & -2.166255 & -2.959626 \\
\hline 72 & 1 & 0.790306 & -2.403072 & -3.962790 \\
\hline 73 & 1 & 2.100984 & -0.613148 & -2.949543 \\
\hline 74 & 6 & 1.128871 & 5.758593 & -0.446343 \\
\hline 75 & 1 & 1.349100 & 6.765886 & -0.107752 \\
\hline 76 & 6 & 1.508587 & 3.916509 & -1.970623 \\
\hline 77 & 6 & 1.805416 & 5.204968 & -1.539379 \\
\hline 78 & 1 & 2.027883 & 3.503651 & -2.829687 \\
\hline 79 & 1 & 2.563055 & 5.782107 & -2.062274 \\
\hline
\end{tabular}

$\mathrm{HF}=-2050.9363879$ Hartree

Zero-point correction $=0.669208$

Sum of electronic and thermal Free Energies = -2050.336194 
(I)- $\left[\mathrm{Pb}\left(\mathrm{L}^{10}-\mathrm{H}\right)\right]^{+}$

(0 imaginary frequencies)

\begin{tabular}{|c|c|c|c|c|}
\hline \multirow{2}{*}{$\begin{array}{l}\text { Center } \\
\text { Number }\end{array}$} & \multirow{2}{*}{$\begin{array}{l}\text { Atomic } \\
\text { Number }\end{array}$} & \multicolumn{3}{|c|}{ Coordinates (Angstroms) } \\
\hline & & $\mathrm{X}$ & $\mathrm{Y}$ & $\mathrm{Z}$ \\
\hline 1 & 1 & 0.734232 & -1.208541 & -4.079729 \\
\hline 2 & 1 & 0.258529 & -2.859883 & -3.697201 \\
\hline 3 & 1 & -2.077961 & -2.066718 & -3.701579 \\
\hline 4 & 1 & 1.948385 & 0.257869 & -3.257661 \\
\hline 5 & 1 & 2.560553 & -2.564040 & -3.051713 \\
\hline 6 & 6 & 0.535873 & -1.897251 & -3.248847 \\
\hline 7 & 1 & 3.632019 & -0.193855 & -3.051764 \\
\hline 8 & 6 & -1.781866 & -2.075775 & -2.642150 \\
\hline 9 & 1 & 0.372909 & 1.329710 & -2.271202 \\
\hline 10 & 1 & -1.656071 & -3.128493 & -2.349902 \\
\hline 11 & 6 & 2.724226 & 0.105167 & -2.499235 \\
\hline 12 & 6 & 1.776649 & -2.151011 & -2.388029 \\
\hline 13 & 1 & -5.508838 & 3.291120 & -2.034799 \\
\hline 14 & 8 & -0.547397 & -1.380473 & -2.470190 \\
\hline 15 & 1 & -2.979593 & -0.363316 & -2.216693 \\
\hline 16 & 1 & -3.841769 & -1.900617 & -2.120885 \\
\hline 17 & 1 & -3.737307 & 4.822821 & -1.175118 \\
\hline 18 & 1 & 1.509496 & -2.934839 & -1.674579 \\
\hline 19 & 1 & 5.118945 & 1.281923 & -2.041422 \\
\hline 20 & 6 & 2.993427 & 1.408860 & -1.777749 \\
\hline 21 & 6 & -2.896425 & -1.392044 & -1.856683 \\
\hline 22 & 7 & 0.587551 & 1.755443 & -1.371184 \\
\hline 23 & 1 & -0.075293 & 2.511773 & -1.212104 \\
\hline 24 & 6 & -4.713745 & 2.912086 & -1.400297 \\
\hline 25 & 7 & 2.273943 & -1.000178 & -1.614879 \\
\hline 26 & 6 & 4.305819 & 1.874704 & -1.628984 \\
\hline 27 & 6 & -3.724063 & 3.767549 & -0.917427 \\
\hline 28 & 6 & 1.942684 & 2.198551 & -1.267796 \\
\hline 29 & 1 & 4.275883 & -1.675780 & -1.283414 \\
\hline 30 & 1 & -5.465747 & 0.897514 & -1.405945 \\
\hline 31 & 6 & -4.678819 & 1.559978 & -1.052389 \\
\hline 32 & 6 & 4.589811 & 3.072190 & -0.973641 \\
\hline 33 & 6 & 2.224947 & 3.397151 & -0.604153 \\
\hline 34 & 1 & 5.616762 & 3.408382 & -0.871679 \\
\hline 35 & 6 & 3.377979 & -1.381287 & -0.707479 \\
\hline 36 & 1 & 1.407641 & 3.994558 & -0.206975 \\
\hline 37 & 6 & -2.708382 & 3.267612 & -0.102410 \\
\hline 38 & 6 & 3.540750 & 3.830366 & -0.453538 \\
\hline 39 & 1 & -1.930730 & 3.931948 & 0.266910 \\
\hline 40 & 1 & -2.437934 & -3.417311 & -0.433718 \\
\hline 41 & 7 & -2.721032 & -1.330075 & -0.390036 \\
\hline 42 & 1 & 2.953757 & -3.479570 & -0.266906 \\
\hline 43 & 1 & 3.646050 & -0.490462 & -0.132716 \\
\hline 44 & 1 & 3.741089 & 4.765115 & 0.061456 \\
\hline 45 & 6 & -3.670248 & 1.034503 & -0.236982 \\
\hline 46 & 6 & -2.667799 & 1.906297 & 0.250336 \\
\hline 47 & 82 & -0.031778 & -0.351263 & 0.207683 \\
\hline 48 & 1 & -3.921798 & -2.919113 & 0.381389 \\
\hline 49 & 6 & -2.858247 & -2.663449 & 0.236738 \\
\hline 50 & 6 & 3.095749 & -2.526132 & 0.262039 \\
\hline 51 & 1 & -4.744014 & -0.799851 & -0.006969 \\
\hline 52 & 6 & -3.733673 & -0.411222 & 0.207271 \\
\hline 53 & 1 & 3.980534 & -2.645175 & 0.905175 \\
\hline 54 & 7 & -1.621228 & 1.383905 & 1.031757 \\
\hline 55 & 8 & 1.946411 & -2.242786 & 1.052969 \\
\hline 56 & 1 & 1.448793 & -4.247715 & 1.223756 \\
\hline
\end{tabular}




\begin{tabular}{|c|c|c|c|c|}
\hline 57 & 1 & -3.599339 & -0.453513 & 1.292409 \\
\hline 58 & 8 & -0.729546 & -2.758355 & 1.305780 \\
\hline 59 & 6 & -2.131487 & -2.808068 & 1.571866 \\
\hline 60 & 6 & -1.185416 & 2.028812 & 2.095778 \\
\hline 61 & 1 & -1.739455 & 2.892431 & 2.475350 \\
\hline 62 & 6 & 1.508080 & -3.342940 & 1.846449 \\
\hline 63 & 1 & -2.390625 & -3.780670 & 2.014197 \\
\hline 64 & 1 & -2.415536 & -2.027202 & 2.291459 \\
\hline 65 & 6 & 0.133621 & -3.029127 & 2.412805 \\
\hline 66 & 1 & 2.212570 & -3.534860 & 2.669069 \\
\hline 67 & 1 & -0.226769 & -3.901272 & 2.975740 \\
\hline 68 & 1 & 0.170908 & -2.163451 & 3.086576 \\
\hline 69 & 6 & -0.020365 & 1.625915 & 2.774107 \\
\hline 70 & 6 & 0.581649 & 2.133955 & 3.948302 \\
\hline 71 & 6 & 1.728555 & 1.370638 & 4.164695 \\
\hline 72 & 6 & 1.782779 & 0.429649 & 3.116199 \\
\hline 73 & 7 & 0.736307 & 0.570607 & 2.276988 \\
\hline 74 & 1 & 0.213002 & 2.953677 & 4.552409 \\
\hline 75 & 1 & 2.442273 & 1.472523 & 4.971574 \\
\hline 76 & 1 & 2.533354 & -0.330653 & 2.937464 \\
\hline
\end{tabular}

\section{(II)-[Pb( $\left.\left(\mathrm{L}^{10}-\mathrm{H}\right)\right]^{+} \quad$ (0 imaginary frequencies)}

\begin{tabular}{|c|c|c|c|c|}
\hline \multirow{2}{*}{$\begin{array}{l}\text { Center } \\
\text { Number }\end{array}$} & \multirow{2}{*}{$\begin{array}{l}\text { Atomic } \\
\text { Number }\end{array}$} & \multicolumn{3}{|c|}{ Coordinates (Angstroms) } \\
\hline & & $\mathrm{X}$ & $\mathrm{Y}$ & $\mathrm{Z}$ \\
\hline---- & - & -------- & -------- & -------- \\
\hline 1 & 1 & 6.112951 & 0.352291 & -3.227455 \\
\hline 2 & 1 & 4.351848 & 1.679769 & -4.380990 \\
\hline 3 & 1 & 0.850823 & -2.837477 & -2.616334 \\
\hline 4 & 6 & 5.210314 & 0.641089 & -2.697643 \\
\hline 5 & 1 & 1.076965 & -4.443312 & -1.876727 \\
\hline 6 & 6 & 4.223824 & 1.386407 & -3.343061 \\
\hline 7 & 1 & -1.288253 & -4.128809 & -2.282096 \\
\hline 8 & 1 & 3.175070 & -3.482521 & -1.075879 \\
\hline 9 & 6 & 0.661435 & -3.436851 & -1.713152 \\
\hline 10 & 1 & -5.074385 & 1.685479 & -4.026752 \\
\hline 11 & 1 & 2.795750 & -1.810873 & -1.544938 \\
\hline 12 & 1 & 5.819744 & -0.287969 & -0.853557 \\
\hline 13 & 6 & -0.829922 & -3.535814 & -1.475794 \\
\hline 14 & 6 & 5.038194 & 0.273790 & -1.360280 \\
\hline 15 & 6 & 2.650201 & -2.566644 & -0.760646 \\
\hline 16 & 1 & 2.296997 & 2.351064 & -3.145780 \\
\hline 17 & 6 & 3.069524 & 1.766889 & -2.653924 \\
\hline 18 & 1 & -2.851174 & 2.341401 & -3.164971 \\
\hline 19 & 8 & 1.266251 & -2.833732 & -0.576365 \\
\hline 20 & 6 & -4.786038 & 1.406331 & -3.017662 \\
\hline 21 & 8 & -1.367578 & -2.221726 & -1.465645 \\
\hline 22 & 1 & -1.014948 & -4.057468 & -0.524280 \\
\hline 23 & 1 & 4.329677 & -2.064417 & 0.465001 \\
\hline 24 & 1 & -3.280257 & -3.016443 & -1.653518 \\
\hline 25 & 6 & -3.530324 & 1.771720 & -2.534389 \\
\hline 26 & 6 & 3.236558 & -2.111678 & 0.567114 \\
\hline 27 & 1 & -6.661695 & 0.440808 & -2.555996 \\
\hline
\end{tabular}




\begin{tabular}{|c|c|c|c|c|}
\hline 28 & 1 & -3.120922 & -1.258873 & -1.735291 \\
\hline 29 & 6 & 3.885814 & 0.632231 & -0.653626 \\
\hline 30 & 6 & 2.900819 & 1.401028 & -1.316034 \\
\hline 31 & 6 & -2.767342 & -2.147416 & -1.214836 \\
\hline 32 & 1 & 3.030066 & -2.895900 & 1.300312 \\
\hline 33 & 6 & -5.673041 & 0.709146 & -2.197250 \\
\hline 34 & 1 & -1.403828 & 2.478126 & -1.383759 \\
\hline 35 & 6 & -3.137683 & 1.430550 & -1.235247 \\
\hline 36 & 1 & 4.683752 & -0.010005 & 1.233129 \\
\hline 37 & 6 & 3.707150 & 0.275233 & 0.809827 \\
\hline 38 & 7 & 2.715791 & -0.812899 & 1.074085 \\
\hline 39 & 7 & 1.734250 & 1.721460 & -0.577614 \\
\hline 40 & 7 & -1.844372 & 1.802436 & -0.762996 \\
\hline 41 & 6 & -5.275575 & 0.359160 & -0.906943 \\
\hline 42 & 82 & 0.092956 & -0.002980 & 0.117914 \\
\hline 43 & 1 & 2.306708 & 3.687801 & -0.165380 \\
\hline 44 & 6 & -3.107688 & -2.095288 & 0.279106 \\
\hline 45 & 1 & -2.676428 & -2.982533 & 0.752124 \\
\hline 46 & 6 & -4.006387 & 0.683953 & -0.411059 \\
\hline 47 & 6 & 1.624614 & 2.860433 & 0.055828 \\
\hline 48 & 1 & -4.200564 & -2.190585 & 0.393168 \\
\hline 49 & 1 & -5.962452 & -0.187324 & -0.265105 \\
\hline 50 & 1 & 1.364277 & -2.782476 & 2.505864 \\
\hline 51 & 1 & 3.366083 & 1.155429 & 1.361158 \\
\hline 52 & 1 & -1.871680 & 2.220662 & 0.165982 \\
\hline 53 & 1 & 3.321400 & -1.228385 & 3.089269 \\
\hline 54 & 6 & 2.438286 & -0.876133 & 2.529162 \\
\hline 55 & 7 & -2.616975 & -0.901444 & 1.015733 \\
\hline 56 & 6 & 1.262768 & -1.767692 & 2.912416 \\
\hline 57 & 1 & -1.028890 & -2.941529 & 2.107775 \\
\hline 58 & 8 & 0.034221 & -1.193223 & 2.451210 \\
\hline 59 & 6 & -3.604107 & 0.223364 & 0.973012 \\
\hline 60 & 1 & 2.217459 & 0.138917 & 2.873760 \\
\hline 61 & 1 & 1.229057 & -1.845679 & 4.008205 \\
\hline 62 & 6 & -1.101496 & -2.024537 & 2.709456 \\
\hline 63 & 1 & -3.158930 & 1.048161 & 1.542837 \\
\hline 64 & 1 & -4.517787 & -0.072489 & 1.514943 \\
\hline 65 & 6 & -2.385206 & -1.249919 & 2.436224 \\
\hline 66 & 1 & -3.229115 & -1.833400 & 2.843492 \\
\hline 67 & 1 & -1.097774 & -2.318768 & 3.768424 \\
\hline 68 & 1 & -2.340344 & -0.322600 & 3.014313 \\
\hline 69 & 6 & 0.682682 & 3.010293 & 1.103157 \\
\hline 70 & 6 & 0.356578 & 4.130163 & 1.894787 \\
\hline 71 & 6 & -0.541833 & 3.680364 & 2.865638 \\
\hline 72 & 1 & 0.740221 & 5.134938 & 1.769351 \\
\hline 73 & 6 & -0.716397 & 2.303258 & 2.635548 \\
\hline 74 & 1 & -0.995441 & 4.259344 & 3.659127 \\
\hline 75 & 1 & -1.297635 & 1.604926 & 3.224444 \\
\hline 76 & 7 & 0.011843 & 1.883873 & 1.574390 \\
\hline
\end{tabular}

